\title{
Studying the human lower limb perfusion dynamics with the step in place model
}

\author{
Estudo da perfusão dinâmica no membro inferior humano, com o modelo "step in place"
}

Margarida Florindo ${ }^{1,2}$, Sérgio Nuno ${ }^{2,3,4}$, L. Monteiro Rodrigues ${ }^{2,5}$

${ }^{1}$ Escola Superior de Saúde Cruz Vermelha Portuguesa (ESSCVP), Portuguese Red Cross Health School. Área de Ensino de

Fisioterapia, Department of Physiotherapy. Lisboa, Portugal

${ }^{2}$ Universidade Lusófona CBIOS - Research Center for Biosciences and Health Technologies, Av Campo Grande, 376, 1749024, Lisboa, Portugal

${ }^{3}$ Clínica S João de Deus - CTD, Lisboa Portugal

${ }^{4}$ Escola Superior de Tecnologia da Saúde de Lisboa - Instituto Politécnico de Lisboa, ESTeSL Polytechnic Institute of

Lisbon, Av. D. João II, Lote 4.69.01 | 1990-096 Lisboa, Portugal

${ }^{5}$ Universidade de Lisboa, Faculty of Pharmacy, Pharmacol. Sc Depart., Lisboa, Portugal

Email: mflorindo@esscvp.eu

\begin{abstract}
The characterisation of microcirculation can be an important diagnostic instrument in vascular medicine. The aim of this study is to evaluate the impact of the basic movement of walking, under controlled conditions, on the foot microcirculation. Fifteen healthy volunteers $(24.5 \pm 6.5$ years old) both sexes, were selected ( 7 female and 8 male). Under standardised conditions, volunteers were asked to execute a protocol divided into three phases: one minute in a resting standing position (Phase 1); one minute performing a step in place routine (Phase 2); and, one minute to recover back to the resting standing (Phase 3). The blood volume changes were assessed non-invasively by photoplethysmography (PPG) and by polarised spectroscopy (TiVi), and the gastrocnemius activity assessed by surface electromyography (EMG). Results have shown a consistent increase of perfusion related with the proposed activity, where significant higher values of CRBC for men were found in Phases 2 and 3. Further studies are needed to better understand the interest and potential of this model in microcirculatory physiology and pathophysiology.
\end{abstract}

Keywords: Movement and perfusion; microcirculation; blood volume; concentration of red blood cells

\section{Resumo}

A caracterização da microcirculação pode ser um importante instrumento de diagnóstico da área da saúde vascular. O objetivo deste estudo consiste em avaliar o impacto de movimentos simples como caminhar em condições controladas, na circulação periférica do pé. Quinze voluntários saudáveis ( $24.5 \pm 6.5$ anos de idade) de ambos os sexos foram selecionados ( 7 do sexo feminino e 8 do masculino). Os voluntários realizaram um protocolo em condições padronizadas, dividido em 3 fases: 1 minuto na posição de pé em repouso (fase 1); 1 minuto a realizar uma rotina de passo no mesmo local (fase 2) e 1 minuto de recuperação, na posição de pé (fase 3). As alterações no volume sanguíneo foram avaliadas no pé, com os métodos não-invasivos de fotopletismografia (PPG) e espectroscopia polarizada (TiVi). A eletromiografia (EMG) avaliou a atividade muscular dos gastrocnémios. Os resultados evidenciaram um aumento consistente da perfusão relacionado com a atividade proposta, sendo que foram encontrados valores maiores de CRBC no sexo masculino nas fases 2 e 3 . Sugere-se que haja um seguimento deste estudo para continuar a sustentar o potencial deste modelo de avaliação na fisiologia e fisiopatologia da microcirculação.

Palavras chave: movimento e perfusão; microcirculação; volume de sangue; concentração de eritrócitos 


\section{Introdution}

Cardiovascular pathology is differently expressed depending on many patient related determinants, including lifestyles, presence of major risk factors (e.g. diabetes) and sex [1-3]. Human microcirculatory adaptive response is known to be gender dependent [1]. Some studies recognized that males and females of the same species have different metabolic requirements $[1,4]$ but, regarding movement, these studies did not address the importance of foot microcirculation in the gait process and the likely influence of the individual's gender on microvascular physiology. Walking is often referred to as the "sixth vital signal" associated with functional lifestyle and can be used as a rehabilitation instrument to activate the vascular multisystem in the lower limb. Sex related endocrine differences on endothelial function have been assessed [5]. Other studies addressed the relationship between microvascular dysfunction and cardiovascular events often linked to lifestyles based on sedentary habits and inactivity [6-7].

Dysfunctional walking was suggested to decrease gait quality, and consume approximately $40 \%$ more oxygen than normal (walking) individuals [8-10]. Therefore we aim to approach the impact of a basic movement like walking, mimicked by the "step in place" model, on the foot microcirculation.

\section{Material and Methods}

A convenience sample of fifteen healthy volunteers $(24.5 \pm 6.5$ years old $)$ both sexes, were selected ( 7 female and 8 male), with a BMI of $23.0 \pm 2.6$ and a normal ankle-brachial index $(1.10 \pm 0.14)$ corresponding to a normal peripheral circulatory physiology. All procedures were carried out in accordance with the Declaration of Helsinki and respective amendments, observing good clinical practice for medical research Involving human subjects [11].

All the experiments took place in the same room and after 10 minutes of acclimatization in standing. The applied protocol included three phases - one minute in a standing position (resting, Phase 1), one minute performing a step in place routine (Phase 2) and, one minute to recover standing in the same position as in Phase 1 (Phase 3). Local microcirculatory perfusion was quantified in both feet by light based systems, using wavelengths between $440 \mathrm{~nm}$ and $530 \mathrm{~nm}$. Local changes in blood volume (BV) and pulse rate (PR)

\section{Introdução}

A expressão da patologia cardiovascular depende de diferentes indicadores clínicos relacionados com o paciente, incluindo o seu estilo de vida e a presença de importantes fatores de risco (por exemplo, diabetes) e de diferenças de resposta adaptativa dependentes do sexo [1-3]. Alguns estudos reconhecem que homens e mulheres têm requisitos metabólicos diferentes [1,4], contudo, em relação ao movimento, não é abordada a importância da microcirculação do pé no processo da marcha e a provável influência do sexo do indivíduo na fisiologia microvascular. A marcha é frequentemente referida como o "sexto sinal vital", associado ao estilo de vida funcional e pode ser utilizada como um instrumento de reabilitação para ativação do multi-sistema vascular no membro inferior. As diferenças endócrinas relacionadas ao sexo na função endotelial têm sido avaliadas [5], assim como outros estudos abordaram a relação entre disfunção microcirculatória e eventos cardiovasculares frequentemente associados a estilos de vida a hábitos sedentários e de inatividade [6-7]. A marcha disfuncional é uma marcha de qualidade reduzida com compensações que consomem ao indivíduo aproximadamente mais $40 \%$ de oxigénio do que aquela que é realizada em padrões normais [8-10]. Com base nestes pressupostos, este estudo pretende abordar o impacto de um movimento semelhante à marcha, mimetizado pelo modelo de "step in place", na microcirculação do pé.

\section{Material e Métodos}

Foram selecionados quinze voluntários saudáveis $(24,5$ $\pm 6,5$ anos de idade) de ambos os sexos ( 7 do sexo feminino e 8 do masculino), com Índice de Massa Corporal (IMC) de 23,0 \pm 2,6 e Índice Tornozelo-Braço normal $(1,10 \pm 0,14)$, correspondendo a uma fisiologia circulatória periférica normal. Todos os procedimentos foram realizados de acordo com a Declaração de Helsínquia e respetivas alterações, observando as boas práticas clínicas para pesquisas médicas que envolvem seres humanos [11].

Todas as experiências ocorreram na mesma sala e após 10 minutos na posição de pé para aclimatação. O protocolo utilizado incluiu 3 fases - um minuto na posição de pé (repouso, fase 1), um minuto realizando a rotina de passos no mesmo local (fase 2) e, um minuto de recuperação na mesma posição da fase 1 (fase 3). A perfusão microcirculatória local foi quantificada em ambos os pés por sistemas baseados em luz, usando comprimentos de onda entre $440 \mathrm{~nm}$ e $530 \mathrm{~nm}$. As alterações 
were measured through a reflection Photoplethysmography (PPG) sensor placed $1 \mathrm{~cm}$ proximal from the 1st metatarsus phalangeal joint. The concentration of red blood cells (CRBC) was assessed with the Tissue Viability Image system (WheelsBridge, SE) by a portable probe placed as near as possible to the PPG sensor and analysed with the TiVi Wheels Bridge Software. Additionally, to assess the electrical activity contribution of the muscle pump, the surface Electromyography (EMG) was applied to the gastrocnemius muscle. Both PPG sensor and EMG electrodes were attached to a BITalino plugged microprocessor board (PLUX Wireless Biosignals, Portugal), and analysed with MatLab [12]. The blood volume was calculated with PPG signals and expressed as the mean waveform amplitude (arbitrary units - AU). Pulse rate was identified as the number of waveforms per minute (/min). The EMG was expressed as the ratio of the root mean square (rms) of second and third phases, relative to the Phase 1 . The Wilcoxon signed-rank test was used for phase comparisons for each variable and the Mann-Whitney test was used for sex comparison within the same phase. A $p>0.05$ significance level was adopted.

\section{Results and Discussion}

Results are presented in Table 1 and for blood volume values, no significant difference between sexes $(p=0.336)$ could be detected in Phase 1 meaning all volunteers were equivalent in terms of their microcirculatory perfusion baseline. When we compared means of Phase 1 with Phase 3, significant increases in blood perfusion $(p=0.002)$ and pulse rate were found for all subjects, again no difference between sexes. These results do not meet the gender dependence of perfusion described and also in accordance with our own experience [13-16]. Nevertheless, we should note that all these measurements were performed in the standing position, while in the other studies, measurements were taken seated or in supine.

Perfusion measured by PPG was not possible to obtain in Phase 2, as the foot impact on the floor created many artefacts. In turn, variations found in gastrocnemius contraction during all three phases (Figure 1) were expected and, were analysed in relation to the microcirculation variations (see below).

To calculate the mean TiVi index in the same area, obtaining the subsequent images, a region of interest (ROI) was selected and analysed. For CRCB during phase 1 (baseline data) (Figure 1), we found no sex- locais no volume sanguíneo (VS) e na taxa de pulso (FP) foram medidas através de um sensor de fotopletismografia de reflexão (FPG) posicionado a $1 \mathrm{~cm}$ proximal da $1^{\mathrm{a}}$ articulação metatarso-falângica. A concentração de glóbulos vermelhos (CGVS) foi avaliada com o sistema Tissue Viability Image (WheelsBridge, SE) utilizando uma sonda portátil colocada o mais próximo possível do sensor FPG e os dados analisados com o TiVi Wheels Bridge Software. Para avaliar a contribuição da atividade elétrica da bomba muscular, a eletromiografia de superfície (EMG) foi aplicada ao músculo gastrocnémio. O sensor FPG e os elétrodos EMG foram conectados a uma placa de microprocessador da BItalino (PLUX Wireless Biosignals, Portugal) e analisados com o sistema MatLab [12]. O volume sanguíneo foi calculado com sinais PPG e expresso como a amplitude média da forma de onda (unidades arbitrárias - AU).

A frequência de pulso foi identificada como o número de ondas por minuto (/ $\mathrm{min})$. O EMG foi expresso como a razão entre o quadrado médio da raiz (rms) da segunda e terceira fases, em relação à fase 1 . O teste de postos sinalizados de Wilcoxon foi usado para cada variável na comparação entre fases e o teste de Mann-Whitney para a comparação entre valores de cada sexo na mesma fase. Foi adotado o nível de significância p>0,05.

\section{Resultados e Discussão}

Nos resultados apresentados na tabela 1 podemos verificar que nos valores de volume sanguíneo (FPG) não foram observadas diferenças significativas entre os dois sexos $(\mathrm{p}=0,336)$ na fase 1 , o que significa que todos os voluntários foram equivalentes em termos de linha de base de perfusão microcirculatória. Quando comparadas as médias da fase 1 com a fase 3 , foram encontrados aumentos significativos no volume sanguíneo ( $\mathrm{p}$ $=0,002$ ) e na frequência de pulso para todos os indivíduos, novamente sem diferença entre os sexos. Estes resultados não atendem à dependência de perfusão de gênero descrita noutras experiências [13-16]. No entanto, devemos salientar que neste estudo, todas essas medidas foram realizadas na posição de pé, enquanto nos demais estudos as medidas foram realizadas na posição de sentado ou em decúbito dorsal, o que pode dar origem a esta divergência. Não foi possível obter a perfusão medida pelo FPG na fase 2, uma vez que o impacto do pé no chão criou muitos artefactos. Por sua vez, as variações encontradas na contração do gastrocnémio durante todas as três fases (Figure 1) eram esperadas e foram analisadas em relação às variações da microcirculação (veja em parágrafo abaixo).

Para calcular a média do índice de TiVi na mesma área, 
related differences $(\mathrm{p}=0.189)$. A significant decrease of mean CRBC (TiVi-mean index) was detected when comparing Phase 1 and 2, with values consistently higher in male $(p=0.009)$. A similar significant decrease between Phase 1 and 3 was detected for CRBC values ( $p=0.004)$. As published previously, light exercise seems to lower the submaximal blood flow after a period of training [5]. A significant increase in EMG gastrocnemius activity $(p=0.001)$ was detected during Phase 2, but no sex-related differences are found (Table 1). Variations on the lower limb microcirculation might be related to the main driving force of the peripheral circulation determined by the calf muscle pump, as previously suggested $[5,10,17]$.

Comparing $\mathrm{CRBC}$ values from Phase 1 and Phase 3 still shows significant higher differences for male $(\mathrm{p}=0.029)$ affecting all means $(\mathrm{p}=0.036)$, suggesting a quick recovery with higher values in Phase 3. Comparing the EMG values between Phases 1 and 3 we find that the muscle is still activated, suggesting same muscle activity in the recovery phase that can influence the microcirculation. The calf muscle, the gastrocnemius in particular, is important for the lower limb microcirculation homeostasis triggering a complex regulatory feedforward mechanism that changes local blood flow according with task [18-20].

Our results suggest that this simple task can induce a similar effect to walking, and for that it might be also interesting as a rehabilitation exercise. Nevertheless, further studies are necessary to confirm some of the views here expressed, including to increase the " $n$ ", to compare results in different positions, and to compare healthy individuals with different pathophysiological characteristics. foi selecionada e analisada uma região de interesse (ROI) em todas as imagens subsequentes. Para CGVS durante a fase 1 (dados de base iniciais) (Figura 1), não foram encontradas diferenças relacionadas ao sexo $(\mathrm{p}=$ 0,189 ). Uma diminuição significativa dos valores médios dos CGVS (índice médio de TiVi) foi detetado, quando comparadas as fases 1 e 2 , com valores consistentemente mais altos no sexo masculino $(\mathrm{p}=0,009)$. Uma diminuição significativa semelhante entre as fases 1 e 3 foi verificada para os valores de CGVS ( $p=$ $0,004)$. Conforme publicado anteriormente, o exercício ligeiro parece diminuir o fluxo sanguíneo submáximo após um período de treino [5]. Um aumento significativo na atividade dos gastrocnémios foi detetado com EMG durante a fase $2(p=0,001)$, mas não foram encontradas diferenças relacionadas com o sexo (Tabela 1). Variações na microcirculação do membro inferior podem estar relacionadas à principal força motriz da circulação periférica determinada pela bomba muscular da região posterior da perna, como sugerido anteriormente $[5,10,17]$.

A comparação dos valores de CGVS entre as fase 1 e 3 mostra também diferenças significativamente maiores no sexo masculino $(\mathrm{p}=0,029)$, afetando todas as médias $(\mathrm{p}=0,036)$ e sugerindo uma rápida recuperação com valores mais altos na fase 3 . Comparando os valores EMG entre as fases 1 e 3 identificámos que o músculo se mantem significativamente ativo, o que sugere que, a manutenção da atividade muscular na fase de recuperação pode ainda estar a influenciar a microcirculação. Os músculos da bomba muscular periférica, em particular os gastrocnémios, são importante para a homeostasia da microcirculação dos membros inferiores, desencadeando um complexo mecanismo regulador de feedforward que influencia o fluxo sanguíneo local de acordo com a tarefa [18-20].

Os resultados deste estudo sugerem que uma tarefa simples pode induzir um efeito semelhante à marcha $\mathrm{e}$, por isso, também pode ser interessante como exercício de reabilitação. No entanto, novos estudos são necessários para confirmar algumas das opiniões aqui expressas, o que inclui aumentar o " $n$ ", comparar resultados em diferentes posturas e comparar indivíduos saudáveis com diferentes características fisiopatológicas. 
Table 1 / Tabela 1 - Mean and standard deviation of the PPG amplitude and ratios and the EMG recorded in each phase of the protocol ( $\mathrm{F}=$ female; $\mathrm{M}=$ Male; $\mathrm{F}+\mathrm{M}=$ female+male; * $\mathrm{p}<0.05)$. / Média e desvio padrão da amplitude da FPG e rácios, EMG, registados em cada fase do protocolo $\left(\mathrm{F}=\right.$ feminino; $\mathrm{M}=$ Masculino; $\mathrm{F}+\mathrm{M}=$ feminine+masculino; $\left.{ }^{*} \mathrm{p}<0.05\right)$.

\begin{tabular}{|c|c|c|c|c|c|c|c|c|c|c|}
\hline \multirow[b]{2}{*}{$\begin{array}{c}\text { Sex / Sexo } \\
F(n=5) \\
M(n=6)\end{array}$} & & \multicolumn{3}{|c|}{ Phase / Fase 1} & \multicolumn{3}{|c|}{ Phase / Fase 2} & \multicolumn{3}{|c|}{ Phase / Fase 3} \\
\hline & & $\mathrm{F}$ & M & $\mathrm{F}+\mathrm{M}$ & $\mathrm{F}$ & $\mathrm{M}$ & $\mathrm{F}+\mathrm{M}$ & $\mathrm{F}$ & M & $\mathrm{F}+\mathrm{M}$ \\
\hline \multirow{3}{*}{ PPG(AU) / FPG (UA) } & $\begin{array}{l}\text { mean / } \\
\text { média }\end{array}$ & 22 & 23 & 22,5 & \multirow{3}{*}{\multicolumn{3}{|c|}{ N/A }} & 27,3 & 27,0 & 27,1 \\
\hline & $s d / d p$ & 1,38 & 4,88 & 3,13 & & & & 6,1 & 5,1 & 5,6 \\
\hline & $\mathrm{p}$ & \multicolumn{2}{|c|}{0.336} & - & & & & \multicolumn{2}{|c|}{0.536} & $0.002 *$ \\
\hline \multirow{3}{*}{$\begin{array}{c}\text { CRBC (AU) / CCVS } \\
\text { (UA) }\end{array}$} & $\begin{array}{l}\text { mean / } \\
\text { média }\end{array}$ & 247,2 & 299,4 & 273,3 & 225,2 & 284,3 & 254,7 & 230,1 & 294,0 & 262,0 \\
\hline & $s d / d p$ & 17,3 & 66,07 & 41,7 & 11,7 & 52,6 & 32,1 & 16.12 & 66,7 & 66,69 \\
\hline & $\mathrm{p}$ & \multicolumn{2}{|c|}{0.189} & - & \multicolumn{2}{|c|}{$0.009 *$} & $0.004^{*}$ & \multicolumn{2}{|c|}{$0.029 *$} & $0.036 *$ \\
\hline \multirow{3}{*}{$\mathrm{PR} / \mathrm{FP}$ (/min) } & $\begin{array}{l}\text { mean / } \\
\text { média }\end{array}$ & 74 & 65,6 & 69,8 & \multirow{3}{*}{\multicolumn{3}{|c|}{ N/A }} & 76,1 & 71,1 & 73,6 \\
\hline & $s d / d p$ & 3.32 & 10,05 & 10,05 & & & & 2,96 & 11,95 & 7,46 \\
\hline & $\mathrm{p}$ & \multicolumn{2}{|c|}{$0.014 *$} & - & & & & \multicolumn{2}{|c|}{0.463} & $0.001 *$ \\
\hline \multirow{3}{*}{ EMG (rms) ratio } & $\begin{array}{l}\text { mean / } \\
\text { média }\end{array}$ & 1 & 1 & 1 & 1,95 & 2,83 & 2,39 & 1,21 & 1,58 & 1,40 \\
\hline & $s d / d p$ & 0 & 0 & 0 & 0,52 & 1,60 & 1,06 & 0,33 & 0,66 & 0,50 \\
\hline & $\mathrm{p}$ & & & - & & & $0.001^{*}$ & \multicolumn{2}{|c|}{0.152} & $0.001 *$ \\
\hline
\end{tabular}

(a)

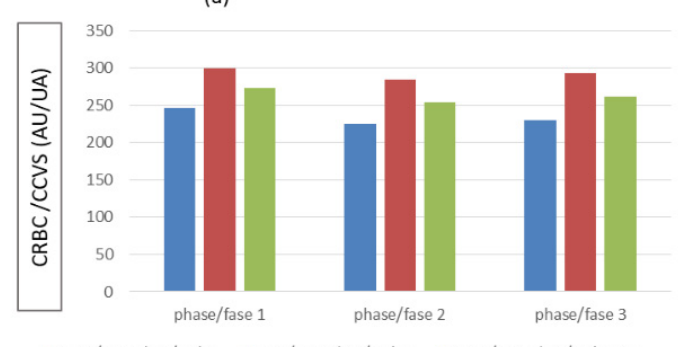

- CRBC/CCVS (AU/UA) F -

(b)

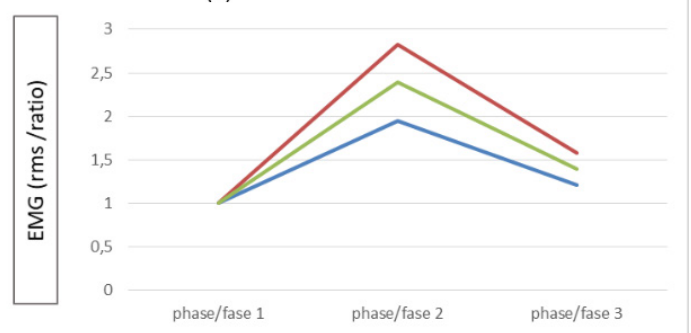

- $\operatorname{sEMG}(\mathrm{rms}) \mathrm{F} \quad \mathrm{sEMG}(\mathrm{rms}) \mathrm{M} \quad \mathrm{sEMG}(\mathrm{rms}) \mathrm{F}+\mathrm{M}$

Figure 1/ Figura 1 - Comparison of CRBC values (a) and EMG ratio (b) between sexes and between total averages during the three phases of the protocol $(\mathrm{F}=$ female; $\mathrm{M}=$ Male; $\mathrm{F}+\mathrm{M}=$ female + male; $* \mathrm{p}<0.05)$.

Comparação dos valores de CGVS (a) e racio de EMG (b) entre sexos e entre a média total durante as três fases do protocolo ( $\mathrm{F}=$ feminino; $\mathrm{M}=$ Masculino; $\mathrm{F}+\mathrm{M}=$ feminine+masculino; $* \mathrm{p}<0.05$ ). 


\section{Acknowledgements}

The authors would like to express their thanks to all the volunteers for their participation in this study. This work was supported by national funds from FCT, within the project UID/DTP/04567/2019.

\section{Conflict of Interests}

All authors declared no conflict of interests.

\section{Agradecimentos}

Os autores gostariam de expressar seus agradecimentos a todos os voluntários por sua participação neste estudo. Este trabalho foi apoiado por fundos nacionais da FCT, dentro do projeto UID/DTP/04567/2019.

\section{Conflito de Interesses}

Os autores não declararam qualquer conflito de interesses 


\section{References /Referências}

1. Kao WL, Sun CW. Gender-Related Effect in Oxygenation Dynamics by Using Far-Infrared Intervention with Near-Infrared Spectroscopy Measurement: A Gender Differences Controlled Trial. PLoS ONE 2015: 10(11): e0135166. doi:10.1371/journal. pone.0135166

2. Margaryants NB, Sidorov IS, Volkolv MV, Gurov IP, Mamontov OV, Kamshilin AA. Visualization of skin capillaries with moving red blood cells in arbitrary area of the body. Vol. 10, No. 9 / 1 September 2019 / Biomedical Optics Express 4896. https://doi.org/10.1364/BOE.10.004896

3. Sara JD, Taher R, Kolluri N Vella A Lerman LO, Lerman A. Coronary microvascular dysfunction is associated with poor glycemic control amongst female diabetics with chest pain and non-obstructive coronary artery disease. Cardiovasc Diabetol (2019) 18:22. https://doi.org/10.1186/s12933-0190833-1

4. Huxley VH, Kem SS. Sex-Specific Characteristics of the Microcirculation. Adv Exp Med Biol. 2018 ; 1065: 307-328. doi:10.1007/978-3-319-779324_20.

5. Glieman L, Hansen CV, Rytter N, Hellsten Y. Regulation of skeletal muscle blood flow during exercise. Current Opinion in Physiology 2019, 10:146-155https://doi.org/10.1016/j.cophys.2019.05.001

6. Leopold JA. Microvascular Dysfunction: Genetic Polymorphisms Suggest Sex-Specific Differences in Disease Phenotype. Coron Artery Dis. 2014 June; 25(4): 275-276. doi:10.1097/MCA.0000000000000122.

7. Vranish JR, Young BE, Stephens BY, Kaur J, Padilla Jaume, Fadel PJ. Brief periods of inactivity reduce leg microvascular, but not macrovascular, function in healthy young men. Experimental Physiology. 2018;103:1425-1434. DOI: 10.1113/EP086918

8. McGowan CP, Neptune RR, Clark DJ, Kautz SA. Modular control of human walking: Adaptations to altered mechanical demands. Journal of biomechanics.2010 Feb 10;43(3):412-9

9. Truijen J, Kim YS, Krediet CTP, Stok WJ, Ko lgen RS, Secher NH, van Lieshout JJ. Orthostatic leg blood volume changes assessed by near-infrared spectroscopy. Exp Physiol 97.3 (2012) pp 353-361. DOI: 10.1113/expphysiol.2011.061051.

10. Caldwell J, Wardlow G, Branch P, Ramos M, Black C, Ade C. Effect of exercise-induced muscle damage on vascular function and skeletal muscle microvascular deoxygenation. Physiol Reports, 2016,4 (22), e13032.

11. World Medical Association. Declaration of Helsinki: ethical principles for medical research involving human subjects. JAMA 310, 2191-2194, https:// doi.org/10.1001/jama.2013.281053 (2013).

12. Vest AN, Poian GD, Li Q, Liu C, Nemati S, Shah AJ, Clifford GD. An Open Source Benchmarked Toolbox for Cardiovascular Waveform and Interval Analysis. Physiol Meas; 39(10): 105004. doi:10.1088/1361-6579/aae021.

13. McDermott MM, Fried L, Simonsick E, Ling S, Guralnik JM. Asymptomatic peripheral arterial disease is independently associated with impaired lower extremity functioning: the women's health and aging study. Circulation. 2000; 101:1007-1012.

14. Silva, H., Bento, M., Vieira, H. \& Rodrigues, L. M. Comparing the spectral components of laser Doppler flowmetry and photoplethysmography signals for the assessment of the vascular response to hyperoxia. Biomed Biopharm Res. (14) 2, 187-194. https://doi.org/10.19277/bbr.14.2.161 (2017).

15. Silva, H., Ferreira, H. A., da Silva, H. P. \& Rodrigues, L. M. The Venoarteriolar Reflex Significantly Reduces Contralateral Perfusion as Part of the Lower Limb Circulatory Homeostasis in vivo. Front Physiol. 9, 1123, https://doi.org/10.3389/fphys.2018.01123 (2018).

16. Rocha, C., Macedo, A., Nuno, S., Silva, H., Ferreira, H. \& Rodrigues, L. M. Exploring the perfusion modifications occurring with massage in the human lower limbs by non-contact polarized spectroscopy. Biomed Biopharm Res. , 2018; (15) 2: , 196-204. DOI: 10.19277/bbr.15.2.186

17. Recek C. Calf pump activity influencing venous hemodynamics in the lower extremity. Int J Angiol. 2013 Mar; 22(1):23-30.

18. Tschakovsky ME, Matusiak K, Vipond C, McVicar L. Lower limb-localized vascular phenomena explain initial orthostatic hypotension up on standing from squat. Am J Physiol Heart Circ Physiol. 2011 Nov; 301(5):H2102-12. doi: 10.1152/ajpheart.00571.2011.

19. Florindo M, Silva H, Monteiro Rodrigues. Impact of the isometric contraction of the calf on the local microcirculation Biomedical Sciences/Ciências Biomédicas Biomed Biopharm Res. 2017; (14) 2: 179-186. DOI: 10.19277/bbr.14.2.160

20. Sinkler SY, Segal SS. Rapid versus slow ascending vasodilatation: intercellular conduction versus flow-mediated signalling with tetanic versus rhythmic muscle contractions. J Physiol 595.23 (2017) pp 7149-7165. DOI: 10.1113/JP275186 